\title{
An aptamer-based shear horizontal surface acoustic wave biosensor with a CVD-grown single- layered graphene film for high-sensitivity detection of a label-free endotoxin
}

\author{
Junwang Ji', Yiquan Pang ${ }^{2}$, Dongxiao $\mathrm{Li}^{1}$, Zheng Huang ${ }^{3}$, Zuwei Zhang ${ }^{4}$, Ning Xue ${ }^{5}, \mathrm{Yi} \mathrm{Xu}^{1}$ and Xiaojing Mu${ }^{1}$
}

\begin{abstract}
The thickness of the sensitive layer has an important influence on the sensitivity of a shear horizontal surface acoustic wave (SH-SAW) biosensor with a delay-line structure and lower layer numbers of graphene produce better sensitivity for biological detection. Therefore, a label-free and highly sensitive SH-SAW biosensor with chemical vapor deposition (CVD-)-grown single-layered graphene (SLG) for endotoxin detection was developed in this study. With this methodology, SH-SAW biosensors were fabricated on a $36^{\circ} \mathrm{Y}-90^{\circ} \mathrm{X}$ quartz substrate with a base frequency of $246.2 \mathrm{MHz}$, and an effective detection cell was fabricated using acrylic material. To increase the surface hydrophilicity, chitosan was applied to the surface of the SLG film. Additionally, the aptamer was immobilized on the surface of the SLG film by cross-linking with glutaraldehyde. Finally, the sensitivity was verified by endotoxin detection with a linear detection ranging from 0 to $100 \mathrm{ng} / \mathrm{mL}$, and the detection limit (LOD) was as low as $3.53 \mathrm{ng} / \mathrm{mL}$. In addition, the stability of this type of SH-SAW biosensor from the air phase to the liquid phase proved to be excellent and the specificity was tested and verified by detecting the endotoxin obtained from Escherichia coli (E. coli), the endotoxin obtained from Pseudomonas aeruginosa (P. aeruginosa), and aflatoxin. Therefore, this type of SH-SAW biosensor with a CVD-grown SLG film may offer a promising approach to endotoxin detection, and it may have great potential in clinical applications.
\end{abstract}

\section{Introduction}

Endotoxins are complex lipopolysaccharides (LPS) that form the cell walls of various gram-negative bacteria. Structurally, LPS consists of lipid A, core polysaccharide, and O-polysaccharide side chains. Among them, lipid A is the main component of the bacterial endotoxin, which determines its toxicity, the O-polysaccharide side chain is highly variable among different bacteria, and the

\footnotetext{
Correspondence: Ning Xue (xuening23xn@163.com) or Yi Xu (xuyibbd@cqu. edu.cn) or Xiaojing Mu (mxjacj@cqu.edu.cn)

${ }^{1}$ Key Laboratory of Optoelectronic Technology \& Systems, Ministry of Education, International R \& D Center of Micro-nano Systems and New Materials Technology, Chongqing University, 400044 Chongqing, China ${ }^{2} \mathrm{~S} c h o o l$ of Chemistry and Chemical Engineering, Chongqing University, 400030 Chongqing, China

Full list of author information is available at the end of the article.
}

specificity determines the serotype of bacteria. Endotoxins are responsible for the toxic effects that cause fevers, ${ }^{1}$ septic shock, ${ }^{2}$ and sepsis. ${ }^{3,4}$ Biosensor based endotoxin detection has been widely investigated recently, ${ }^{5,6}$ and many methods have been investigated to identify endotoxins: hydrophobic interactions, ${ }^{7}$ localized surface plasmon resonance (LSPR), ${ }^{8}$ mass spectrometry, ${ }^{9}$ optical methods,${ }^{10}$ the electronic tongue ${ }^{11}$ the voltammetric method, ${ }^{12}$ and electrochemistry. ${ }^{13-17}$ However, these methods have some disadvantages, such as high costs, a long processing time and the requirement of labeled markers. Thus, the demand for rapid, simple operations and low costs is increasing.

Shear horizontal surface acoustic wave (SH-SAW) biosensors have been widely reported owing to aspects such

\section{(c) The Author(s) 2020}

(c) (i) Open Access This article is licensed under a Creative Commons Attribution 4.0 International License, which permits use, sharing, adaptation, distribution and reproduction in any medium or format, as long as you give appropriate credit to the original author(s) and the source, provide a link to the Creative Commons license, and indicate if changes were made. The images or other third party material in this article are included in the article's Creative Commons license, unless indicated otherwise in a credit line to the material. If material is not included in the article's Creative Commons license and your intended use is not permitted by statutory regulation or exceeds the permitted use, you will need to obtain permission directly from the copyright holder. To view a copy of this license, visit http://creativecommons.org/licenses/by/4.0/. 
as their high selectivity and/or sensitive detection of deoxyribonucleic acid (DNA), ${ }^{18}$ proteins, ${ }^{19}$ and cells. ${ }^{20-23}$ SH-SAW biosensors have the advantages of low cost, operational simplicity, and high sensitivity, and these biosensors can be used in label-free and real-time monitoring. Additionally, SH-SAW biosensors are especially suitable for biological detection in a liquid phase, as the particle displacement is parallel to the SAW propagation direction. Nanogold film have traditionally been used as sensitive layers for these biosensors. In contrast to these materials, the thickness of nanogold films is usually in the range of tens of nanometers, which would undoubtedly affect the performance of a biosensor.

Compared to noble $(\mathrm{Au})$-based electrode materials, carbon-based materials show some advantages. That is, the presence of the $\mathrm{sp}^{2}$ hybridized carbon atom center in their structural backbone provides a route for surface modification. Sensing devices produced with bare-carbonbased materials were able to determine analytes down to trace levels. Additionally, its use as the sensing material could enhance the signal in biosensors. Moreover, graphene is exceptionally biocompatible if used as a sensitive layer for the immobilization of biomolecules. ${ }^{24}$ Therefore, graphene-related materials have recently attracted great interest from researchers in the biosensor field. ${ }^{25}$ Among them, graphene oxide (GO) is widely applied as the sensitive layer of the biosensor. ${ }^{26-31}$ However, in the case of $\mathrm{GO}$, the sensitivity strongly depends on the lateral size, the layer number, and the number of oxygen-containing groups. $^{32}$

The rapid development of the chemical vapor deposition (CVD) technique in recent years has enabled the production of large-area, high-quality graphene films with good structural controllability, which is particularly beneficial for the fabrication of sensing devices. ${ }^{33}$ Biosensors fabricated with graphene have been widely investigated for the detection of viruses, ${ }^{34}$ bacteria, ${ }^{35}$ proteins, ${ }^{36}$ and nucleic acids. ${ }^{37}$ In addition, SLG-based biosensors have demonstrated excellent biocompatibility, conductivity and high sensitivity. ${ }^{38,39}$ Recently, researchers have used a finite element analysis (FET) DNA biosensor with SLG to reach a detection limit of $10 \mathrm{fM}$ for target DNA with a dynamic range of $10 \mathrm{fM}$ to $100 \mathrm{pM}^{40}$ indicating that lower layer numbers of graphene produce better sensitivity for the biosensor. A Hall effect biosensor with ultraclean gold-transferred SLG for the detection of DNA hybridization could increase the concentrations of target or onebase mismatched DNA from $1 \mathrm{pM}$ to $100 \mathrm{nM} .^{41}$ Thus far, SAW-based biosensors for the detection of an endotoxin with SLG have not yet been reported.

In this work, we present a highly sensitive and specific SH-SAW biosensor with SLG film in the sensitive area; the SLG was transferred onto a $36^{\circ} \mathrm{Y}-90^{\circ} \mathrm{X}$ quartz substrate using polymethyl methacrylate (PMMA) after CVD growth. In addition, the aptamer was immobilized on the sensitive area by glutaraldehyde (GA) cross-linked chitosan (CS). ${ }^{42-44}$ The LPS applied in this study was obtained from E. coli 055:B5 (L4524), which was extracted by benzene. Finally, the performance of the biosensor was proven to linearly detect an endotoxin in a wide range from 0 to $100 \mathrm{ng} / \mathrm{mL}$, and the biosensor exhibited a detection limit of $3.53 \mathrm{ng} / \mathrm{mL}$. In contrast, a recent report ${ }^{45}$ on a fluorescent aptamer-based probe for the determination of the LPS of Gram-negative bacteria exhibited a detection limit of $8.7 \mathrm{ng} / \mathrm{mL}$. In addition, the selectivity was verified by distinguishing the endotoxin from endotoxin obtained from P. aeruginosa and aflatoxin, and the stability proved to be excellent. Overall, this type of detection strategy may have great potential in future applications.

\section{Materials and methods \\ Materials and reagents}

The copper foils (purity: 99.8\%) were purchased from Shenzhen Changda Sheng Electronics Co., Ltd. The PMMA was obtained from Wenzhou Yuanteng Plastic Co., Ltd. The $36^{\circ} \mathrm{Y}-90^{\circ} \mathrm{X}$ quartz substrates with $\mathrm{Au}$ (purity: 99.9999\%) were obtained from Wuxi Haoda Electronics Co., Ltd. The hydrochloric acid, acetone, CS, GA and etching solution $\left(\mathrm{FeCl}_{3} / \mathrm{HCl}\right)$ were purchased from Chongqing Xingguanghuabo Company. The aptamer $\left(\mathrm{NH}_{2}-5^{\prime}\right.$-CTT CTG CCC GCC TCC TTC C- TAG CCG GAT CGC GCT GGC CAG ATG ATA TAA AGG GTC AGC CCC CCA -GGA GAC GAG ATA GGC GGA CAC $\mathrm{T}-3^{\prime}$ ) was synthesized by Bioengineering (Shanghai) Co., Ltd.

\section{Fabrication of the SH-SAW biosensor}

The SH-SAW biosensor with a delay-line structure was fabricated with a typical MEMS process, ${ }^{46}$ including lithography development, vacuum magnetron sputtering, and lift-off on a $36^{\circ} \mathrm{Y}-90^{\circ} \mathrm{X}$ quartz substrate with a thickness of $0.5 \mathrm{~mm}$ and a diameter of four inches. A Cr/ $\mathrm{Au}$ film $(40 / 100 \mathrm{~nm})$ was sputtered over the entire wafer surface by magnetron sputtering, and then the IDTs and electrodes were formed by immersing the wafer into acetone to lift off the excess metal. To increase the adhesion of $\mathrm{Au}, \mathrm{Cr}$ was used as an adhesion layer.

\section{The growth and transfer of high-quality single-layered graphene}

The SLG film was synthesized with CVD on copper foil. Prior to growth, the copper foil was thoroughly cleaned with hydrochloric acid and cut into a size of $6 \mathrm{~mm} \times$ $3 \mathrm{~mm}$. The copper foil was placed in a tube furnace and the gas path was kept sealed. The quartz tube was vacuumed and then hydrogen gas was passed into the tube to keep it at atmospheric pressure. The air in the 


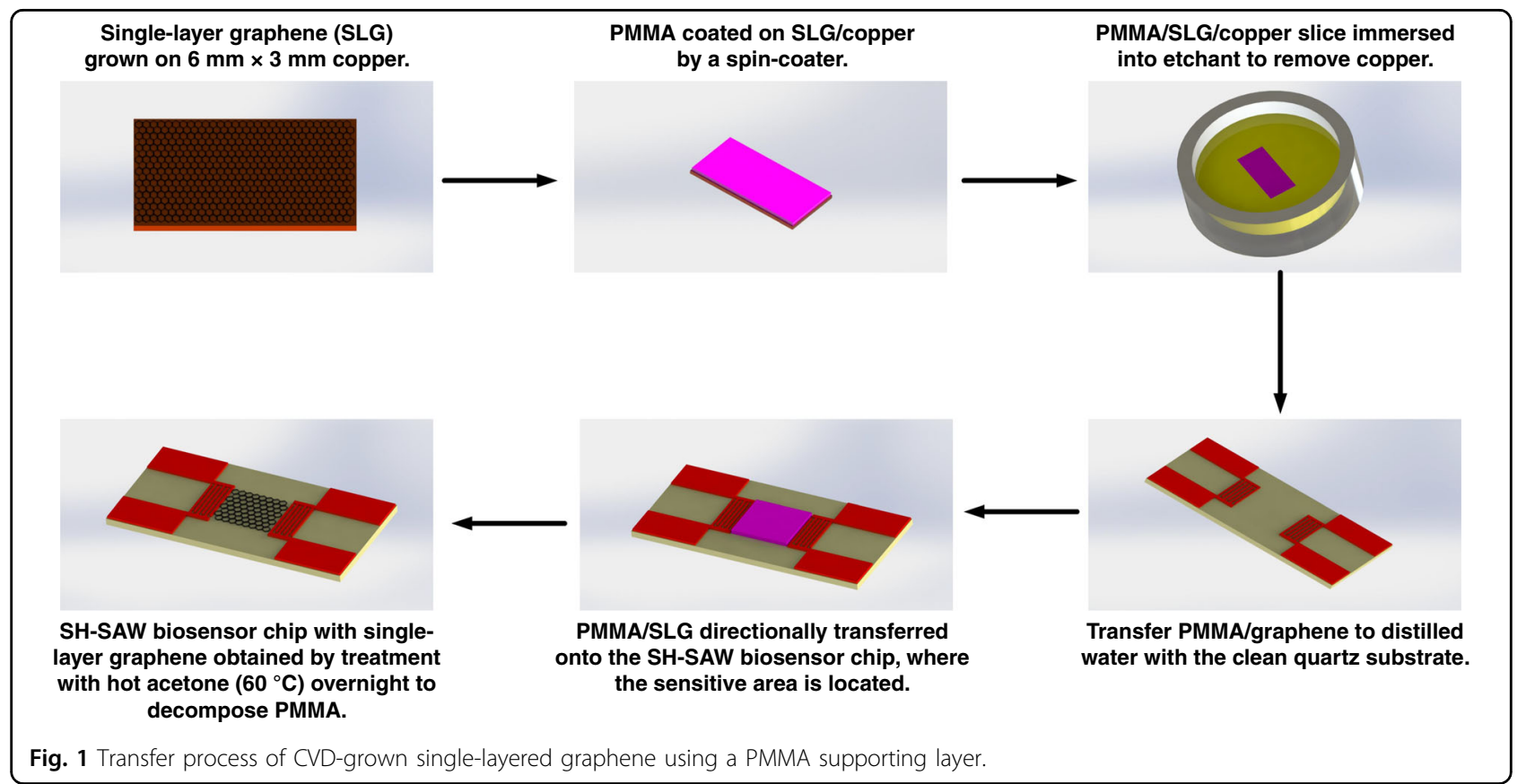

airway was exhausted three times to keep the vacuum pump working continuously with constant pressure $(100 \mathrm{~Pa})$. The system was heated to $1050^{\circ} \mathrm{C}$ in $100 \mathrm{~min}$ with a flow of $\mathrm{H}_{2}(20 \mathrm{sccm})$, and then kept under those conditions for $10 \mathrm{~min}$. Next, a flow of $\mathrm{CH}_{4}$ (35 sccm) was introduced into the system, which was kept under this condition for $20 \mathrm{~min}$, and graphene was grown on the surface of the copper foil. Finally, the chamber was naturally cooled down to room temperature under an $\mathrm{H}_{2} / \mathrm{Ar}$ atmosphere, and then the gas was closed off.

\section{Transfer of high-quality single-layered graphene using PMMA}

As shown in Fig. 1, prior to the transfer of graphene on the quartz substrate, one side of the copper foil was treated with plasma to clean up the graphene. This action was performed to increase the ease of corrupting the copper foil in the later stage. Next, a layer of polymethyl methacrylate (PMMA) was spin-coated on the other side of the copper foil. After the PMMA layer was baked, the copper foil was placed on the surface of the $\mathrm{FeCl}_{3} / \mathrm{HCl}$ etching solution. Half an hour later, the graphene/PMMA was transferred to distilled water for two hours to clean the surface impurities when the copper foil was corroded. After rinsing with deionized water, a clean glass plate was used to support the PMMA/graphene layer $(6 \mathrm{~mm} \times$ $3 \mathrm{~mm}$ in area). After baking at $60^{\circ} \mathrm{C}$ for $2 \mathrm{~h}$, the sample was immersed in hot acetone $\left(60^{\circ} \mathrm{C}\right)$ for $10 \mathrm{~min}$ to dissolve the PMMA followed by annealing in the CVD furnace under a $\mathrm{H}_{2} / \mathrm{Ar}(20 / 80 \mathrm{sccm})$ atmosphere at $450{ }^{\circ} \mathrm{C}$ for $2 \mathrm{~h}$ to remove the rest of the PMMA. High-quality single-layered graphene was then obtained. Finally, for the sensitive layer, the single-layered graphene was transferred onto the sensitive area of the $36^{\circ} \mathrm{X}-90^{\circ} \mathrm{Y}$ quartz substrate to characterize the performance of the biosensor.

\section{Related theory}

In this study, a delay line structure with a single channel was designed, which generally consists of input and output IDTs that were mounted on the $36^{\circ} \mathrm{Y}-90^{\circ} \mathrm{X}$ quartz substrate. The SH-SAW is simulated by the input IDTs, and then propagates along the surface of the sensitive area through the delay line which lies between the input IDTs and the output IDTs. The SH-SAW resonant frequency can be calculated by the following equation:

$$
f_{0}=V_{\mathrm{s}} / 2(a+b)
$$

where $f_{0}$ is the resonant frequency of the device $(\mathrm{MHz}), V_{\mathrm{s}}$ is the SH-SAW propagating velocity on the piezoelectric substrate $(\mathrm{m} / \mathrm{s}), a$ is the width of the IDT fingers and $b$ is the gap between the IDT fingers $(\mu \mathrm{m})$. Generally, a simple situation, namely, $\lambda=a=b$ is adopted; thus, $f_{0}$ can be further expressed as

$$
f_{0}=V_{s} / 4 \lambda
$$

The amplitude and the phase velocity varied with the change in the mass loading on the propagation path. The phase change was collected by the detection system. The phase change and the amplitude change are expressed as 
follows

$$
\begin{aligned}
& \Delta \mathrm{Ph}=360 f l\left(\frac{1}{V_{0}}-\frac{1}{V_{1}}\right), \\
& \Delta \mathrm{Amp}=f l\left(\frac{a_{1}}{V_{1}}-\frac{a_{0}}{V_{0}}\right),
\end{aligned}
$$

where $\Delta \mathrm{Ph}$ is the phase shift of the SH-SAW biosensor $\left({ }^{\circ}\right)$, $f$ is the frequency $(\mathrm{Hz}), l$ is the propagation length $(\mathrm{m}), V_{0}$ is the velocity before loading $(\mathrm{m} / \mathrm{s}), V_{1}$ is the velocity after loading $(\mathrm{m} / \mathrm{s}), \Delta \mathrm{Amp}$ is the amplitude change of the $\mathrm{SH}$ SAW biosensor $(\mathrm{d} B), a_{0}$ is the propagation loss before loading $(\mathrm{d} B / \lambda)$, and $a_{1}$ is the propagation loss after loading $(\mathrm{d} B / \lambda)$.

\section{Immobilization of the aptamer on single-layered graphene}

Prior to the immobilization of the aptamer, the CS solution $(0.2 \%)$ was dropped on the surface of the sensitive area and dried at room temperature to increase the hydrophilicity of the SLG film. Next, $5 \mu \mathrm{L}$ of GA solution in acetic acid at $2.5 \%(\mathrm{~m} / \mathrm{m})(50 \mathrm{mmol} / \mathrm{L}, \mathrm{PH}=7.4)$ was dropped onto the surface of the modified sensitive area, and then the sensitive area was soaked in a GA solution for $2 \mathrm{~h}$ to link the amino-groups in the CS. After that, the SH-SAW biosensor was rinsed with deionized water to clean the residual reagent, and then dried at room temperature. The aptamer solution $(10 \mathrm{nmol} / \mathrm{L})$ was then pumped into the reaction cell, so that the aptamer could be chemically bonded to the aldehyde-groups in the GA. The sensitive area was then washed with deionized water to remove the unreacted aptamer. After that, the endotoxin solution $(0-100 \mathrm{ng} / \mathrm{mL})$ was pumped into the reaction cell, and then the aptamer would specifically bind to the endotoxin. Therefore, the endotoxin could be detected by an SH-SAW biosensor with a CVDgrown SLG.

\section{Detection procedures}

Prior to the experiment, a homemade detection cell was fabricated to increase the ease of operation (Fig. S1). SMA cables were used to connect the vertical network analyzer (Agilent, E5080A) (Fig. S1D), and the phase signals were monitored and recorded by a biosensor monitoring system based on the LabVIEW software. First, the surface of the sensitive area was treated with CS and GA to ensure the linking of the related groups, and then the detection biosensor chip was put into the groove of the detection cell. The phase $\left(P_{0}\right)$ of the SH-SAW biosensor was then monitored until a steady baseline was observed in the liquid phase environment. Next, the aptamer solution was pumped into the reaction cell to link the aldehyde in GA until the phase reached a stable value, and the steady-state phase was taken as $P_{1}$. Finally, the endotoxin solution was pumped into the reaction cell, and the biosensor was then maintained at $37^{\circ} \mathrm{C}$ for the reaction. When the resonance phase reached a stable value, the steady-state phase was taken as $P_{2}$. The phase shift attributed to the aptamerendotoxin reaction was calculated by the equation: $\Delta P=P_{2}-P_{1}$.

\section{Selectivity assessment}

To assess the selectivity, SH-SAW biosensor chips were employed to detect aflatoxin and the endotoxin obtained from Pseudomonas aeruginosa (P. aeruginosa). The aptamer was immobilized on the sensitive surface of the SH-SAW biosensor chip, and then the aflatoxin and the endotoxin obtained from $\mathrm{P}$. aeruginosa were injected into the reaction cells to verify the selectivity.

\section{Electrical measurements and characterizations}

Detailed information regarding the electrical measurements and the characterizations of the equipment used in this study are given in SI-1.2.

\section{Results and discussion}

The principles and the structure of the SH-SAW biosensor with SLG

The detailed configuration of the SH-SAW device with SLG is shown in Fig. 2. The SLG was deposited on the sensitive area of the SH-SAW device as the sensitive layer (Fig. 2a). Additionally, the real product of the SH-SAW biosensor with the SLG is shown in Fig. 2b. The specific parameters of the SH-SAW device are shown in SI-2.1. To illustrate this approach for the high sensitivity detection of the endotoxin, the principle of the SH-SAW biosensor is demonstrated in Fig. 2c, d. The aptamer was first immobilized on the SLG film, and then the endotoxin was captured based on the specific interaction between the aptamer and the endotoxin. The phase shift of the SHSAW biosensor was thus observed for the mass-change in the sensitive area. Additionally, the propagation characteristics of the SH-SAW is verified by the COMSOL 5.2a software (Fig. S3).

The aptamer immobilization processes are shown in Fig. 3. The CS was first immobilized on the SLG film, and then the amino groups in the CS reacted with the aldehyde in $\mathrm{GA}$ to form $\mathrm{C}=\mathrm{N}$ bonds. After that, the aldehydes groups in GA reacted with the amine-functionalized aptamer. It was then ready for the specific detection of an endotoxin.

\section{Characterizations of SLG}

The Raman spectra of the SLG film are presented in Fig. 4a. The Raman spectra exhibit the characteristic peak of a high-quality SLG: a sharp G-band $\left(\approx 1600 \mathrm{~cm}^{-1}\right)$ and a sharp 2D-band $\left(2699-2720 \mathrm{~cm}^{-1}\right) .{ }^{47}$ The $I_{2 \mathrm{D}} / I_{\mathrm{G}}$ ratio (2.37) shows the excellent quality of the SLG. 


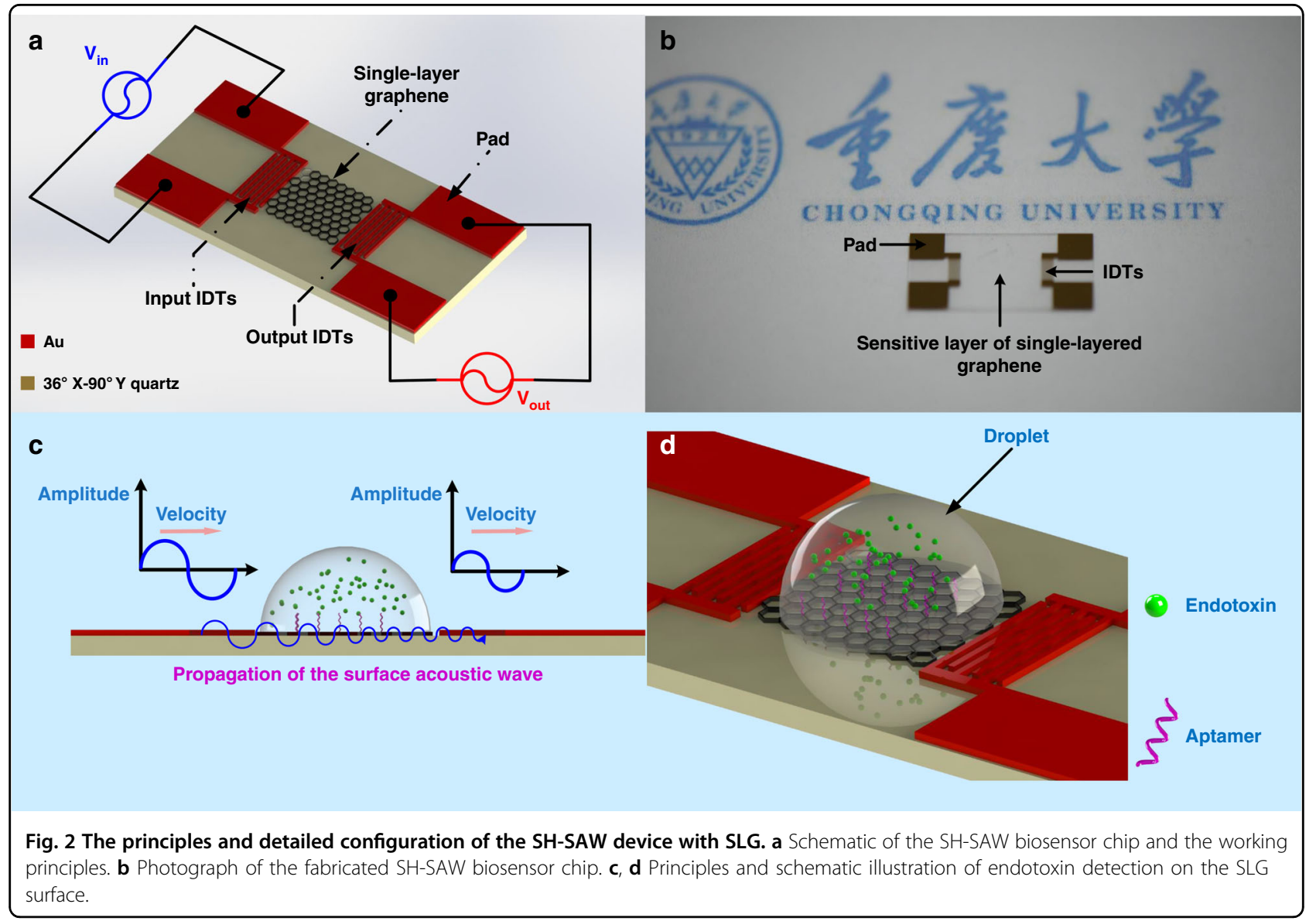

To examine and analyze the surface chemical compositions of the SLG, X-ray photoelectron spectroscopy (XPS) was conducted. As shown in Fig. 4b, the blue line represents the raw spectrum. To fit the $\mathrm{C} 1 \mathrm{~s}$ spectrum, four components were obtained: the $\mathrm{sp}^{2}$ component of $\mathrm{C}=\mathrm{C}$ (green line, at $284.6 \mathrm{eV}$ ) and the oxygencontaining groups including $\mathrm{C}-\mathrm{O}$ (purple line, at $286.1 \mathrm{eV}$ ), $\mathrm{C}=\mathrm{O}$ (black line, at $287.1 \mathrm{eV}$ ), and $\mathrm{COOH}$ (red line, at $288.7 \mathrm{eV}){ }^{48}$ The contents of the chemical states of these elements at different peak positions were calculated using the peak area. As shown in Fig. 4c-e, the data indicated that impurities could not be found on the surface of the SLG film. Therefore, the PMMA was completely removed from the graphene surface due to hot acetone.

Additionally, scanning electron microscopy (SEM) and transmission electron microscopy (TEM) were used to characterize the surface topography of the sensitive area and the single-layered graphene obtained from the sensitive area respectively. Figure $4 \mathrm{a}$ shows that the black area is the single-layered graphene film grown in the sensitive area by CVD, and the white area is the quartz substrate. It is known that graphene possesses good electrical conductivity; hence, this obvious dividing line (Fig. 5a) indicates that the single-layered graphene kept a certain distance from the IDTs. Therefore, short circuit of the IDTs could be avoided. Figure 5b shows polymers on the surface of the single-layered film. As shown in Fig. 5c, d, the morphology of the single-layered graphene is very clear. To prepare test samples, graphene was obtained from the sensitive area. Next, the samples were made into a suspension by ultrasonic oscillation in alcohol. Finally, the suspension was dropped on ultrathin carbon mesh for testing with TEM.

\section{Characterization by AFM}

The AFM images are presented in Fig. 6. The surface morphology of the sensitive area with single-layered graphene is illustrated in Fig. 6a, b, which show that the surface morphology is very smooth. Figure $6 \mathrm{c}$, d indicate that the surface morphology was remarkably changed after the aptamer was immobilized in the sensitive area. After the endotoxin was pumped into the reaction cell, the obvious change in the surface morphology (Fig. 6e, f) indicated that the specific binding of aptamer and 


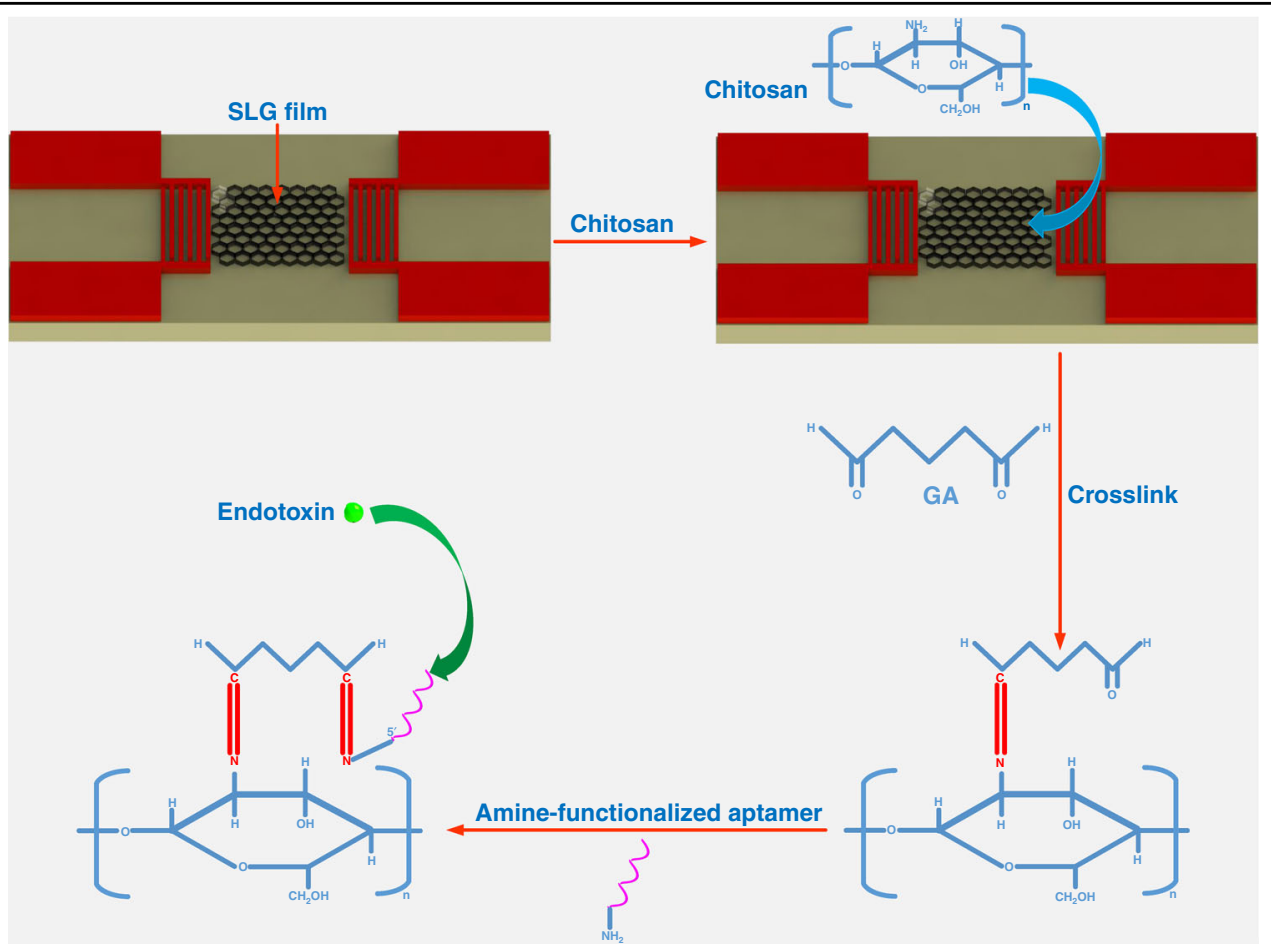

Fig. 3 The surface functionalization process including the surface modification with chitosan and the glutaraldehyde to amine-functionalized aptamer immobilization processes.

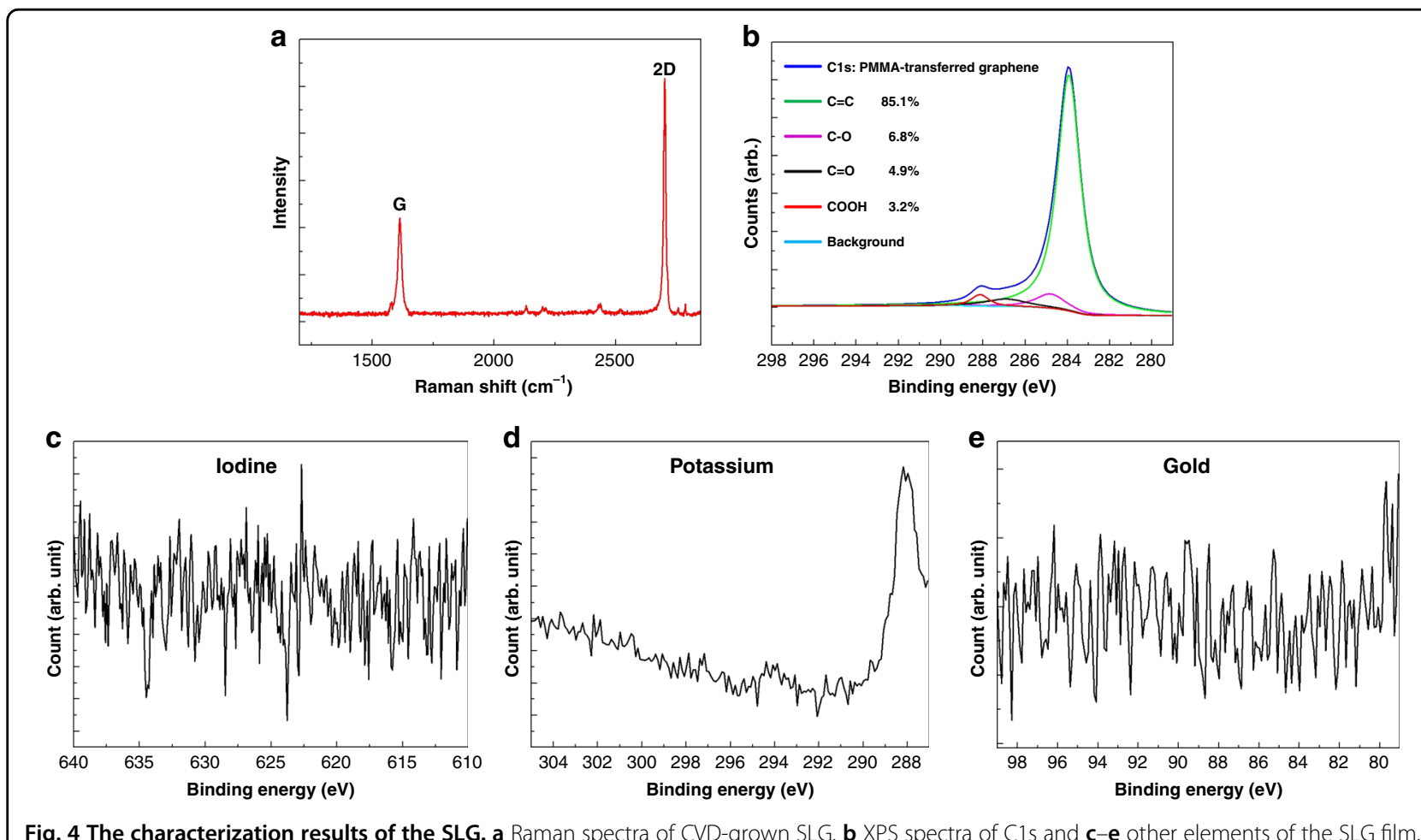

endotoxin occurred in the sensitive area. The parameters used for the AFM technique in the morphological analysis were the following: imaging resolution was 256, the scanning speed was $0.7 \mathrm{~Hz}$, the imaging mode was the tapping mode, and the imaging force setpoint was as follows: 2.9313 P: 1.4 I: 0.7 . 


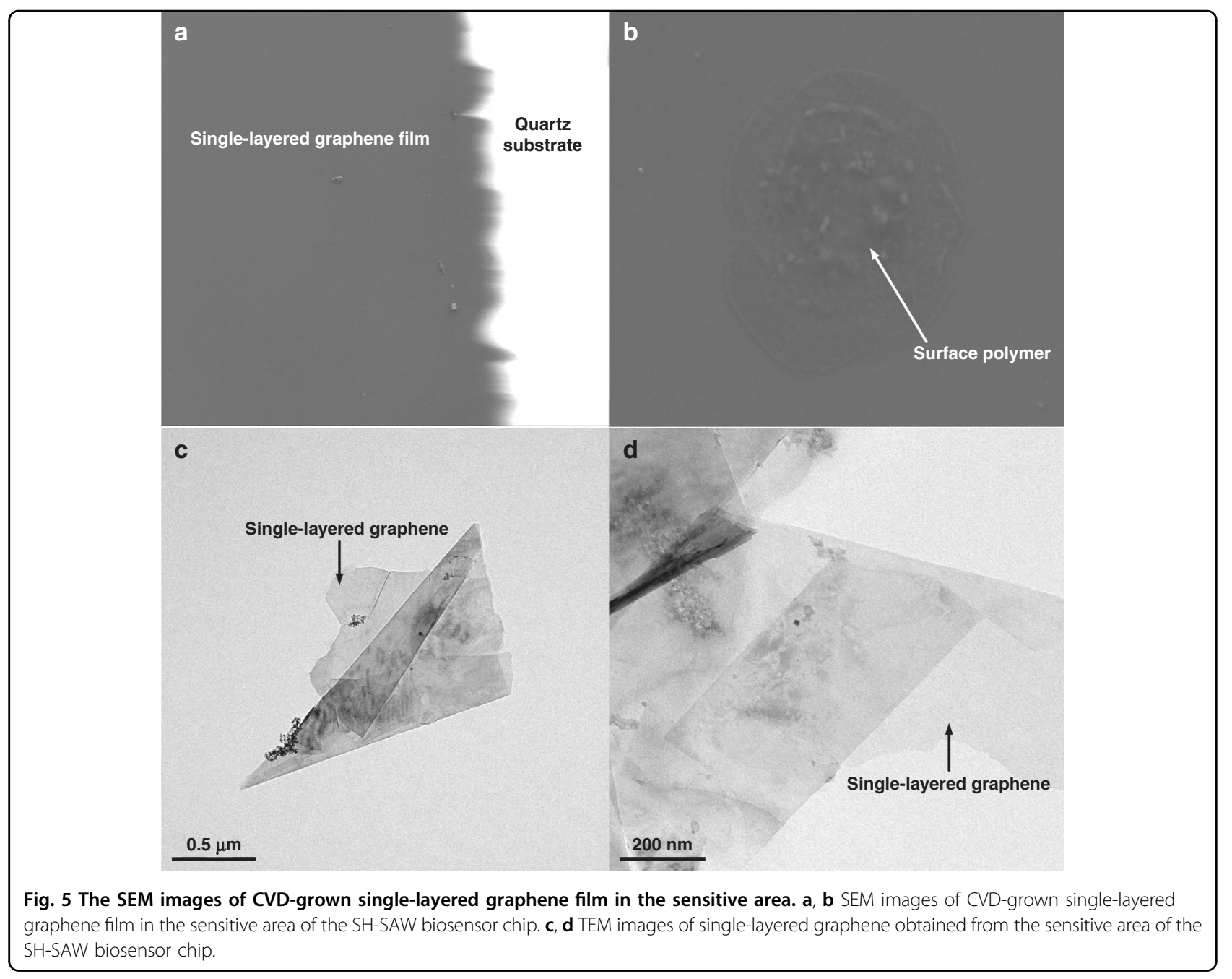

The analytical performance of the SH-SAW biosensor with SLG

Stability is an important factor used to evaluate the performance of an SH-SAW biosensor. Initially, the PBS solution was pumped into the reaction cell while the phase signal was kept stable in the air phase. Then the PBS solution was pumped out of the reaction cell. The result (Fig. 7a) revealed that the phase signal could remain in the stable state when sudden environmental changes occurred. Therefore, the SH-SAW biosensors had excellent stability and external interference could be excluded. The phase shift can be attributed to the mass added to the sensitive area, and thus, the authenticity of the data can be guaranteed. In addition, the endotoxin was pumped into the sensitive area without the aptamer being immobilized on it, which confirms that the phase shift was indeed caused by the specific binding of the aptamer and the endotoxin. The result is shown in Fig. 7b. The phase was kept in the steady state after the endotoxin was pumped into the reaction cell. Therefore, the phase shift can be guaranteed by the specific binding of the aptamer and the endotoxin. In addition, the phase shifts induced by pumping the PBS and aptamer into the sensitive area without the SLG film are shown in Fig. S4. The results revealed that the SLG film was used to immobilize the aptamer in the sensitive area by the crosslinking method.

In the stable test environment, the performance of the SH-SAW biosensor with the SLG film was assessed by detecting the different concentrations of the endotoxin (blank, 10, 25, 50, 75, and $100 \mathrm{ng} / \mathrm{mL}$ ). The real-time phase shifts are presented in Fig. 8a, and the corresponding histogram is shown in Fig. $8 \mathrm{~b}$. As one can see from the results, the phase shifts increased with increasing endotoxins concentration. The phase shifts were linear for the concentration of endotoxin in the range from blank to $100 \mathrm{ng} / \mathrm{mL}$, with a correlation coefficient of 0.97767 (Fig. $8 \mathrm{c})$. In the linear region, the calculated sensitivity was $\mathrm{S} \approx$ $0.044 \mathrm{deg} / \mathrm{ng} / \mathrm{mL}$. The limit of detection was as low as $3.53 \mathrm{ng} / \mathrm{mL}$, which was the blank concentration plus the three-fold standard deviation. The CVs of the $\triangle$ Phase 

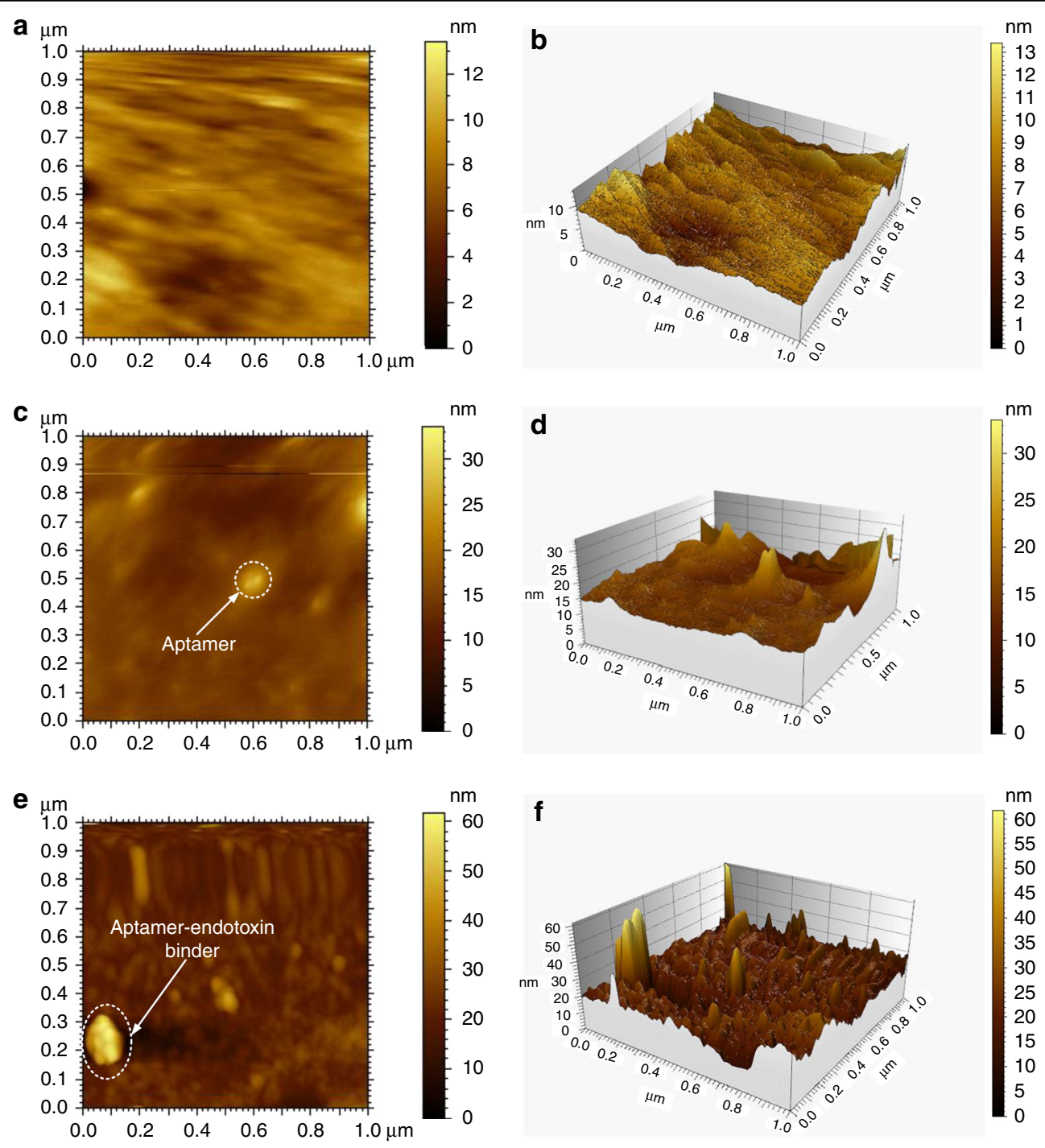

Fig. 6 The AFM images the surface morphology of the sensitive area with single-layered graphene during the experiments. $\mathbf{a}$, $\mathbf{b}$ AFM images of the surface morphology of the sensitive area with single-layered graphene. $\mathbf{c}, \mathbf{d}$ AFM images of the surface morphology of the sensitive area after the aptamer was immobilized. e, f AFM images of the surface morphology of the sensitive area after the specific binding of the aptamer and the endotoxin.

obtained from different concentrations of endotoxin are shown in Table S3. The outcome of the experiment is also compared with some other reported results in Table 1.

The selectivity is especially important to the performance of an SH-SAW biosensor. A nontarget biological sample may cause a higher phase shift than the actual value, which would undoubtedly lead to incorrect experimental results. Therefore, the endotoxin obtained from $\mathrm{P}$. aeruginosa and the aflatoxin were pumped into the reaction cell with a concentration of $50 \mathrm{ng} / \mathrm{mL}$ to test and verify the selectivity of the SH-SAW biosensor. As shown in Fig. 8d, the phase shifts of the endotoxin obtained from P. aeruginosa and the aflatoxin were almost the same as those of the blank solution, while the phase shift of the endotoxin with a concentration of $50 \mathrm{ng} / \mathrm{mL}$ was approximately $3.5^{\circ}$. The results reveal that the $\mathrm{SH}$ SAW biosensor in this study had excellent selectivity in discriminating the endotoxin from the endotoxin obtained from P. aeruginosa and the aflatoxin.

\section{Conclusions}

In this work, we reported a highly sensitive and labelfree method for the detection of an endotoxin by an SH-SAW biosensor with SLG film. This technology proved the high sensitivity of the SH-SAW biosensor, and provided an effective platform for the detection of an endotoxin. The SH-SAW biosensor demonstrated a linear relationship with the concentration range of the 

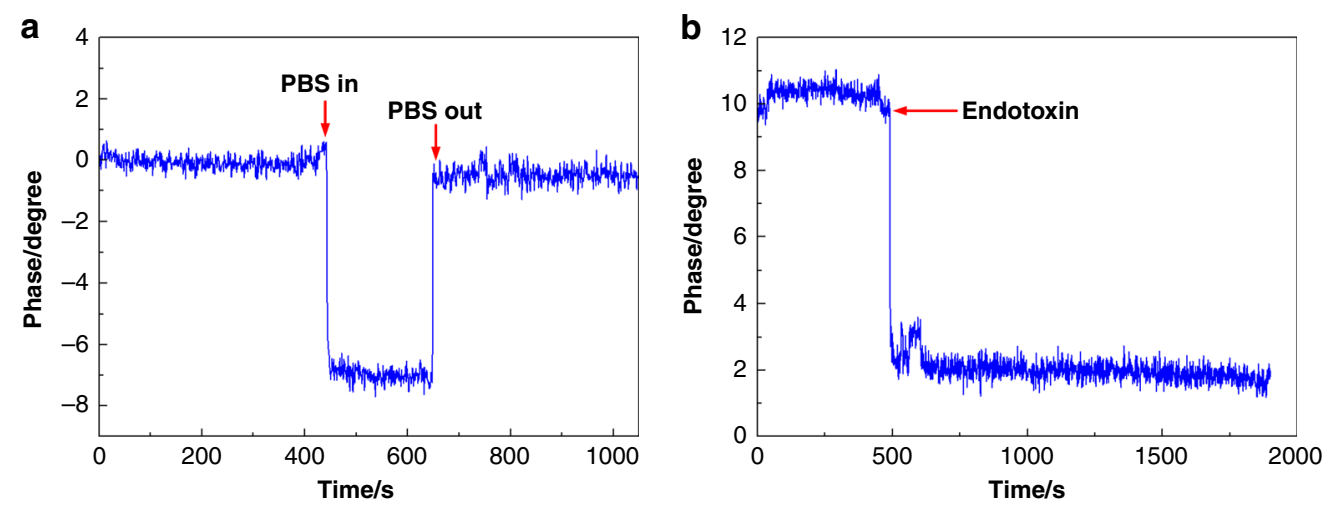

Fig. 7 The stability of the SH-SAW biosenor and the verification of aptamer role. a Phase changes of the SH-SAW biosensor chip in liquid and air, and $\mathbf{b}$ phase shift induced by pumping the endotoxin into the sensitive area without the aptamer immobilized in it.
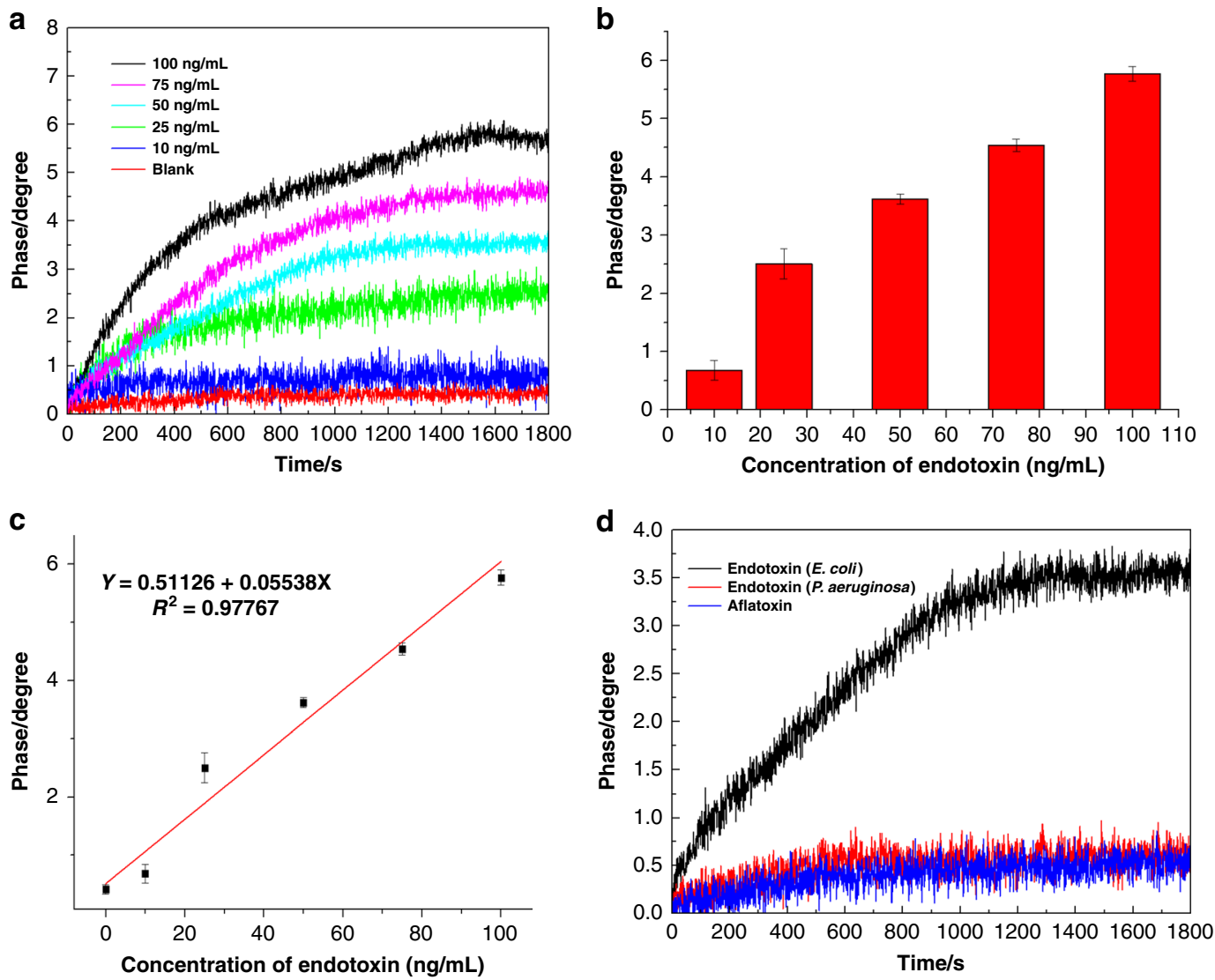

Fig. 8 The sensitivity and selectivity of the SH-SAW biosensor with SLG film. a The real-time monitoring phase shift signals of the specific binding of the aptamer and the endotoxin at different concentrations. $\mathbf{b}$ Histogram of the specific binding of the aptamer to the endotoxin at different concentrations. c Standard curve for the quantitative detection of an endotoxin with an SH-SAW biosensor based on single-layered graphene. The phase shift on the $y$-axis had a linear relationship with the increasing concentrations of endotoxin on the $x$-axis. Each data point represents an average of at least three measurements \pm the standard deviation. $\mathbf{d}$ The real-time phase signals of the endotoxin obtained from E. coli, the endotoxin obtained from P. aeruginosa, and the aflatoxin binding with the aptamer, which were all analyzed at concentrations of $50 \mathrm{ng} / \mathrm{mL}$.

endotoxin from 0 to $100 \mathrm{ng} / \mathrm{mL}$, and a detection limit of $3.53 \mathrm{ng} / \mathrm{mL}$ was achieved. In addition, the stability and excellent specificity make the SH-SAW biosensor a promising alternative to conventional endotoxin detection methods. Therefore, an SH-SAW biosensor with SLG may offer a more effective and accurate prognosis evaluation in clinical diagnosis. However, the repeatability of this device was not ideal. In the future, this biosensor may 
Table 1 Comparison of the sensitivity and the LOD to those of other reported studies.

\begin{tabular}{|c|c|c|c|c|c|}
\hline Method & Material & Detection & Linear range & LOD & Reference \\
\hline Fluorescence quenching & Graphene oxide (GO) & Endotoxin & $10-500 \mathrm{ng} / \mathrm{mL}$ & $8.7 \mathrm{ng} / \mathrm{mL}$ & 46 \\
\hline SH-SAW & Gold & Biotin & $10-400 \mu \mathrm{g} / \mathrm{mL}$ & $<6 \mu \mathrm{g} / \mathrm{mL}$ & 22 \\
\hline SH-SAW & Gold & HIV & $0.75-3 \mu \mathrm{g} / \mathrm{ml}$ & 165 ng/mL & 23 \\
\hline Field effect transistor & CVD graphene & ss-DNA & $1 \mathrm{fM}-1 \mathrm{pM}$ & $10 \mathrm{fM}$ & 41 \\
\hline Love-wave & Gold & Prostate antigen & $10 \mathrm{ng} / \mathrm{mL}-1 \mu \mathrm{g} / \mathrm{mL}$ & $10 \mathrm{ng} / \mathrm{mL}$ & 24 \\
\hline SH-SAW & CVD single-layered graphene & Endotoxin & $0-100 \mathrm{ng} / \mathrm{mL}$ & $3.53 \mathrm{ng} / \mathrm{mL}$ & This work \\
\hline
\end{tabular}

be developed as a miniaturized and versatile device. However, more detailed work should be performed before clinical application.

\section{Acknowledgements}

This study was supported by the National Natural Science Foundation of China (Grant No. 51605060), the National Key Research and Development Program of China (Grant No. 2016YFB0402702), the National Natural Science Foundation of China (Grant No. 81430053), the Army's Logistics Research Program Major Project (Grant No. AWS14C003-2), the Fundamental Research Funds for the Central Universities (No. 2018CDPTCG0001-5), and Chongqing basic science and frontier technology research project (cstc2017jcyjAX0237), and the Chongqing municipality key research and development program of China (Grant No. cstc2017rgzn-zdyfX0041). In addition, we sincerely appreciate the Chongqing Institute of Green and Intelligent Technology, which also assisted in the implementation of this research.

\section{Author details \\ 'Key Laboratory of Optoelectronic Technology \& Systems, Ministry of Education, International R \& D Center of Micro-nano Systems and New Materials Technology, Chongqing University, 400044 Chongqing, China. ${ }^{2}$ School of Chemistry and Chemical Engineering, Chongaing University, 400030 Chongqing, China. ${ }^{3}$ Department of Applied Physics, Chongqing University, 401331 Chongqing, China. ${ }^{4}$ Chongqing Acoustic-Optic-Electric Corporation, China Electronic Technology Group Corporation, 400060 Chongqing, China. ${ }^{5}$ Institute of Electronics, Chinese Academy of Sciences, 100190 Beijing, China}

\section{Conflict of interest}

The authors declare that they have no conflict of interest.

Supplementary information accompanies this paper at https://doi.org/ 10.1038/s41378-019-0118-6.

Received: 13 June 2019 Revised: 10 September 2019 Accepted: 11 October 2019

Published online: 10 February 2020

\section{References}

1. Su, W. et al. Selective detection of endotoxin using an impedance aptasensor with electrochemically deposited gold nanoparticles. Innate Immun. 19, 388-397 (2013).

2. Ding, S., Chang, B., Wu, C., Chen, C. \& Chang, H. A new method for detection of endotoxin on polymyxin B-immobilized gold electrodes. Electrochem. Commun. 9, 1206-1211 (2007)

3. Gutsmann, T. et al. Structural prerequisites for endotoxic activity in the Limulus test as compared to cytokine production in mononuclear cells. Innate Immun. 16, 39-47 (2010).

4. Yaroustovsky, M. et al. Prognostic value of endotoxin activity assay in patients with severe sepsis after cardiac surgery. J. Inflamm. 10, 8 (2013).
5. Das, A. P., Kumar, P. S. \& Swain, S. Recent advances in biosensor based endotoxin detection. Biosens. Bioelectron. 51, 62-75 (2014).

6. Dullah, E. C. \& Ongkudon, C. M. Current trends in endotoxin detection and analysis of endotoxin-protein interactions. Crit. Rev. Biotechnol. 37, 251-261 (2017).

7. Prasad, P., Sachan, S., Suman, S., Swayambhu, G. \& Gupta, S. Regenerative core-shell nanoparticles for simultaneous removal and detection of endotoxins. Langmuir 34, 7396-7403 (2018).

8. Zandieh, M. et al. Label-free and simple detection of endotoxins using a sensitive LSPR biosensor based on silver nanocolumns. Anal. Biochem. 548, 96-101 (2018).

9. Li, H. L., Victoria, M., Hitchins, V. M. \& Wickramasekara, S. Rapid detection of bacterial endotoxins in ophthalmic viscosurgical device materials by direct analysis in real time mass spectrometry. Anal. Chim. Acta 943, 98-105 (2016).

10. Wu, J. C., Zawistowski, A., Ehrmann, M., Yi, T. \& Schmuck, C. Peptide functionalized polydiacetylene liposomes act as a fluorescent turn-on sensor for bacterial lipopolysaccharide. J. Am. Chem. Soc. 133, 9720-9723 (2011).

11. Heras, J. Y., Pallarola, D. \& Battaglini, F. Electronic tongue for simultaneous detection of endotoxins and other contaminants of microbiological origin. Biosens. Bioelectron. 25, 2470-2476 (2010).

12. Liu, T. et al. Preparation of a peptide-modified electrode for capture and voltammetric determination of endotoxin. ACS Omega 2, 2469-2473 (2017).

13. $\mathrm{Su}, \mathrm{W}$. Q. et al. Determination of endotoxin through an aptamer-based impedance biosensor. Biosens. Bioelectron. 32, 32-36 (2012).

14. Fetter, L. et al. Electrochemical aptamer scaffold biosensors for detection of botulism and ricin toxins. Chem. Commun. 51, 15137 (2015).

15. Inoue, K. Y. et al. Development of an electrochemical limulus amebocyte lysate assay technique for portable and highly sensitive endotoxin sensor. Innate Immun. 18, 343-349 (2012).

16. Miao, P. et al. Electrochemical investigation of endotoxin induced limulus amebocyte lysate gel-clot process. Electrochem. Commun. 26, 29-32 (2013).

17. Bai, L. J., Chai, Y. Q., Pu, X. Y. \& Yuan, R. A signal-on electrochemical aptasensor for ultrasensitive detection of endotoxin using three-way DNA junction-aided enzymatic recycling and graphene nanohybrid for amplification. Nanoscale $\mathbf{6}$, 2902 (2014)

18. Hur, Y., Han, J., Seon, J., Pak, Y. E. \& Roh, Y. Development of an SH-SAW sensor for the detection of DNA hybridization. Sens. Actuators A 120, 462-467 (2005).

19. Gizeli, E. et al. Sensitivity of the acoustic waveguide biosensor to protein binding as a function of the waveguide properties. Biosens. Bioelectron. 18, 1399-1406 (2003).

20. Chang, K. et al. Label-free and high-sensitive detection of human breast cancer cells by aptamer-based leaky surface acoustic wave biosensor array. Biosens. Bioelectron. 60, 318-324 (2014).

21. Trivedi, S. \& Nemade, H. B. Coupled resonance in SH-SAW resonator with S1813 micro-ridges for high mass sensitivity biosensing applications. Sens. Actuators B 273, 288-297 (2018).

22. Turbé, $\mathrm{V}$. et al. Towards an ultra-rapid smartphone-connected test for infectious diseases. Sci. Rep. 7, 1-11 (2017).

23. Zhang, F. et al. A microfluidic love-wave biosensing device for PSA detection based on an aptamer beacon probe. Sensors 15, 13839-13850 (2015).

24. Sharma, P. S., D'Souza, F. \& Kutner, W. Graphene and graphene oxide materials for chemo- and biosensing of chemical and biochemical hazards. Top. Curr. Chem. 348, 237-266 (2014). 
25. Suvarnaphaet, P. \& Pechprasarn, S. Graphene-based materials for biosensors: a review. Sensors 17, 2161 (2017).

26. Ye, Y. K. et al. A label-free electrochemical DNA biosensor based on thionine functionalized reduced graphene oxide. Carbon 129, 730-737 (2018).

27. Primo, E. N. et al. Label-free graphene oxide-based surface plasmon resonance immunosensor for the quantification of Galectin-3, a novel cardiac biomarker. ACS Appl. Mater. Interfaces 10, 23501-23508 (2018).

28. Chou, J. C. et al. The flexible urea biosensor using magnetic nanoparticles. IEEE Trans. Nanotechnol. 18, 484-490 (2019).

29. Mogha, N. K., Sahu, V., Sharma, R. K. \& Masram, D. T. Reduced graphene oxide nanoribbon immobilized gold nanoparticle based electrochemical DNA biosensor for the detection of Mycobacterium tuberculosis. J. Mater. Chem. B 6, 5181 (2018).

30. Wang, Y., Zhai, F. G., Hasebe, Y., Jia, H. M. \& Zhang, Z. Q. A highly sensitive electrochemical biosensor for phenol derivatives using a graphene oxidemodified tyrosinase electrode. Bioelectrochemistry 122, 174-182 (2018).

31. Dreyer, D. R., Park, S., Bielawski, C. W. \& Ruoff, R. S. The chemistry of graphene oxide. Chem. Soc. Rev. 39, 228-240 (2010).

32. Muti, M., Sharma, S., Erdem, A. \& Papakonstantinou, P. Electrochemical monitoring of nucleic acid hybridization by single-use graphene oxide-based sensor. Electroanalysis 23, 272-279 (2011).

33. Chen, X. P., Zhang, L. L. \& Chen, S. S. Large area CVD growth of graphene. Synth. Met. 210, 95-108 (2015).

34. Lee, G. J., Choi, C., Kim, D. H. \& Song, Y. M. Bioinspired artificial eyes: optic components, digital cameras, and visual prostheses. Adv. Funct. Mater. 28, 24 (2018).

35. Li, J. et al. Electrochemical immunosensor based on graphene-polyaniline composites and carboxylated graphene oxide for estradiol detection. Sens. Actuators B 188, 99-105 (2013).

36. Yang, S., Zhang, F., Liang, Q. \& Wang, Z. A three-dimensional graphene-based ratiometric signal amplification aptasensor for MUC1 detection. Biosens. Bioelectron. 120, 85-92 (2018).
37. Wasfi, A., Awwad, F. \& Ayesh, A. I. Graphene-based nanopore approaches for DNA sequencing: a literature review. Biosens. Bioelectron. 119, 191-203 (2018).

38. Zheng, $C$. et al. Fabrication of ultrasensitive field-effect transistor DNA biosensors by a directional transfer technique based on CVD-grown graphene. ACS Appl Mater. Interfaces 7, 16953-16959 (2015).

39. Loan, P. T. K. et al. Hall effect biosensors with ultraclean graphene film for improved sensitivity of label-free DNA detection. Biosens. Bioelectron. 99, 85-91 (2018).

40. Lu, Q., Dong, X. C., Li, L. J. \& Hu, X. Direct electrochemistry-based hydrogen peroxide biosensor formed from single-layer graphene nanoplatelet-enzyme composite film. Talanta 82, 1344-1348 (2010).

41. Li, L. L., Peng, J. M., Chu, Z. Y., Jiang, D. F. \& Jin, W. Q. Single layer of graphene/ Prussian blue nano-grid as the low-potential biosensors with high electrocatalysis. Electrochim. Acta 217, 210-217 (2016).

42. Song, J. Y. et al. Selective determination of $\mathrm{Cr}(\mathrm{VI})$ by glutaraldehyde crosslinked chitosan polymer fluorophores. ACS Sens. 3, 792-798 (2018).

43. Hao, N. et al. AgBr nanoparticles/3D nitrogen-doped graphene hydrogel for fabricating all-solid-state luminol-electrochemiluminescence Escherichia coli aptasensors. Biosens. Bioelectron. 97, 377-383 (2017).

44. Tena, S. T. et al. Highly sensitive Escherichia coli shear horizontal surface acoustic wave biosensor with silicon dioxide nanostructures. Biosens. Bioelectron. 93, 146-154 (2017)

45. Wen, L. X. et al. The electrical detection of lead ions using gold-nanoparticleand DNAzyme-functionalized graphene device. Microchimica Acta 186, 122 (2019).

46. Greco, G. et al. Surface acoustic wave (SAW)-enhanced chemical functionalization of gold films. Sensors 17, 2452 (2017).

47. Malard, L. M., Pimenta, M. A., Dresselhaus, G. \& Dresselhaus, M. S. Raman spectroscopy in graphene. Phys. Rep. 473, 51-87 (2009).

48. Poh, H. L. et al. Graphenes prepared by Staudenmaier, Hofmann and Hummers methods with consequent thermal exfoliation exhibit very different electrochemical properties. Nanoscale 4, 3515-3522 (2012). 